\title{
Chemosensitizing Properties of $\beta$-Caryophyllene and $\beta$-Caryophyllene Oxide in Combination with Doxorubicin in Human Cancer Cells
}

\author{
SILVIA DI GIACOMO ${ }^{1}$, ANTONELLA DI SOTTO ${ }^{1}$, GABRIELA MAZZANTI $^{1}$ and MICHAEL WINK $^{2}$ \\ ${ }^{I}$ Department of Physiology and Pharmacology "V. Erspamer", Sapienza University of Rome, Rome, Italy; \\ ${ }^{2}$ Institute of Pharmacy and Molecular Biotechnology - Heidelberg University, Heidelberg, Germany
}

\begin{abstract}
Background/Aim: The natural sesquiterpenes $\beta$ caryophyllene $(C R Y)$ and $\beta$-caryophyllene oxide (CRYO) were evaluated for their potential chemosensitizing properties. Materials and Methods: CRY and CRYO cytotoxicity was tested against the Caco-2, CCRF/CEM and CEM/ADR5000 human cancer cell lines. Furthermore, combination experiments were carried out in order to study the ability of the sesquiterpenes to increase doxorubicin cytotoxicity. The possible interference of CRY and CRYO with functionality of ATP-binding cassette (ABC)transporters was also investigated by Rhodamine 123 efflux assay. Results: Despite a low cytotoxicity, both substances were able to enhance the cytotoxicity of doxorubicin in all cell lines, with CRYO being the most effective reversal agent. Both sesquiterpenes were also able to increase the Rho123 content into cells, likely due to inhibition of the efflux pumps. Conclusion: Our results highlight a potential role of CRY and CRYO as new chemosensitizing agents for doxorubicin chemotherapy and to re-sensitize cancer-resistant cells.
\end{abstract}

Doxorubicin is a widely used chemotherapy approach, due to its ability to suppress the pro-growth signaling in various cancer cells. Unfortunately, the serious side-effects (mainly cardiotoxicity) and the development of multidrug resistance (MDR) limit its clinical application (1). Different mechanisms, particularly the reduced accumulation of anticancer drug into cells, have been found involved in the doxorubicin-mediated MDR. Due to its hydrophobic nature, doxorubicin is easy to

Correspondence to: Antonella Di Sotto, Ph.D. and Silvia Di Giacomo, Ph.D., Department of Physiology and Pharmacology "V. Erspamer”, Sapienza University of Rome, P.le Aldo Moro 5, 00185 Rome, Italy. Tel: +39 0649912904, Fax: +39 0649912480, e-mail: antonella.disotto@uniroma1.it, silvia.digiacomo@uniroma1.it

Key Words: B-caryophyllene, $\beta$-caryophyllene oxide, sesquiterpenes, natural compound, multidrug resistance, doxorubicin. uptake by passive diffusion into cells; conversely, its efflux requires to be mediated by ATP-binding cassette (ABC) transporters, mainly $\mathrm{P}$-glycoprotein (P-gp), multidrug resistance protein 1 (MRP-1) and breast cancer resistance protein (BCRP). Overexpression of the above proteins in cancer cells has been involved in the doxorubicin-mediated resistance (2-4). Therefore, blocking these transporters may increase the intracellular levels of doxorubicin, thus increasing its effectiveness at low doses and reducing its chemotherapy side effects (5). Recently, different compounds have been highlighted able to resensitize cancer MDR cells when administered at low cytotoxic doses in combination with known anticancer drugs, so acting as chemosensitizing agents. However, only few compounds have reached clinical trial experimentation, and none of them has been used in clinical therapy until now (6): this increases the need of searching for new bioactive agents. In recent years, many researchers have turned their attention to natural products because they provide novel chemical scaffolds suitable for the development of new inhibitors. In particular, sesquiterpenes seem to be interesting candidates in combination therapy due to their possible penetration-enhancing properties. Among them, $\beta$ caryophyllene (CRY) and $\beta$-caryophyllene oxide (CRYO) are noteworthy, as several studies have highlighted interesting chemopreventive properties, such as anti-inflammatory, genoprotective and antiproliferative (7).

In this context, the present study aimed to evaluate the potential ability of CRY and CRYO (Figure 1) to act as chemosensitizing agents in order to overcome doxorubicin resistance.

\section{Materials and Methods}

Cell lines. Human colorectal adenocarcinoma Caco-2 (expressing many ABC-transporters, including P-gp, MRP1 and BCRP) and Tcell leukemia CCRF/CEM (expressing low levels of MDR1 transcript) were obtained from Leibniz Institute DSMZ-German Collection of Microorganisms and Cell Cultures (Braunschweig, Germany). CEM/ADR5000 cells (a MDR1-overexpressing and 
A

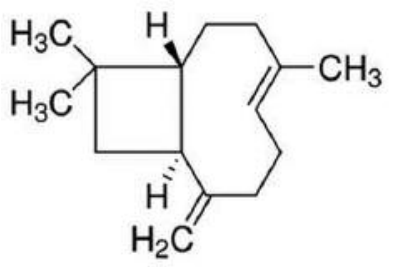

B

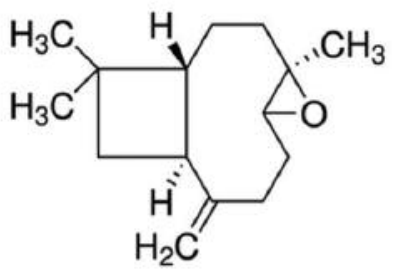

Figure 1. Chemical structure of $\beta$-caryophyllene (A) and $\beta$-caryophyllene oxide $(B)$.

doxorubicin-resistant leukemic subline) were kindly provided by Professor Thomas Efferth (German Cancer Research Center, Heidelberg). All the cell lines were used and cultivated according to the methods described by Di Giacomo et al. (8).

Cytotoxicity. Cell viability was evaluated by the 3-[4,5dimethylthiazol-2-yl]-2,5-diphenyl tetrazolium bromide (MTT) assay (8) and determined as follow: Viability $(\%)=[($ OD treated cells-OD medium control)/(OD untreated cells-OD medium control) $] \times 100$.

Combination assay. Cytotoxicity of doxorubicin (0.00005-1,000 $\mu \mathrm{M})$ was tested by MTT assay, in the presence of CRY or CRYO, at the inhibitory concentration (IC) which induce $10 \%\left(\mathrm{IC}_{10}\right)$ and $20 \%\left(\mathrm{IC}_{20}\right)$ cytotoxicity, as found by the dose-response curve. The type of interaction (synergistic, additive or antagonistic) was evaluated by combination index (CI), isobologram analysis (IB), and reversal ratio value (RR) (8).

Rhodamine 123 efflux assay. Rhodamine 123 (Rho123) is a fluorescent dye effluxed by P-gp and MRP pumps, and accumulated into cells, due to the presence of an $\mathrm{ABC}$ transporter inhibitor. This assay was carried out according to Di Giacomo et al. (8), by using verapamil $(20 \mu \mathrm{M})$ as a standard P-gp and MRP1 inhibitor.

Statistical analysis. GraphPad Prism ${ }^{\mathrm{TM}}$ (Version 4.0) software (GraphPad Software, Inc., San Diego, CA, USA) was used. All data are expressed as mean \pm standard error mean. Each treatment was tested in triplicate in each experiment, which in turn was carried out three times. The one-way analysis of variance (one-way ANOVA), followed by Dunnett's multiple comparison post test, was used to analyze the difference between treatments. The concentrationresponse curves were obtained by Hill equation, according to the software instructions.

\section{Results}

Cytotoxicity. CRY and CRYO significantly reduced cell viability although at much higher concentrations than the positive control doxorubicin (Table I). Among the different cell lines, Caco- 2 was found to be the most resistant cells to the treatment with $\mathrm{CRY}\left(\mathrm{IC}_{50}=1103.34 \pm 10.73 \mu \mathrm{M}\right)$ and $\mathrm{CRYO}$ $\left(\mathrm{IC}_{50}=332.30 \pm 3.97 \mu \mathrm{M}\right)$. In CCRF/CEM and CEM/ADR5000 cell lines the sesquiterpenes showed a similar cytotoxicity profile. The $\mathrm{IC}_{50}$ values were $311.59 \pm 1.44$ and $235.18 \pm 5.18$ in CCRF/CEM, and 368.48 \pm 2.23 and 297.98 \pm 3.33 in CEM/ADR5000 for CRY and CRYO, respectively.

Combination assay. Under our experimental conditions, the co-treatment with both sesquiterpenes generally enhanced the cytotoxicity of doxorubicin in all cell lines, but not always in a statistically significant way (Table I), being CRYO the most effective reversal agent. In Caco- 2 cell line, the combination with CRYO at $\operatorname{IC}_{10}(100 \mu \mathrm{M})$ and $\mathrm{IC}_{20}(248 \mu \mathrm{M})$ determined a statistically significant $(p<0.01$ and $p<0.001)$ decrease of the $\mathrm{IC}_{50}$ value of doxorubicin (Figure 2A, Table I) of about 2- and 4.7-fold, respectively. The isobologram analysis was in agreement with the combination index and highlighted a synergistic effect of the sesquiterpenes on the anticancer drug (Figure 2A). Analogously, in the sensitive CCRF/CEM cell line, the co-incubation of doxorubicin with $\operatorname{IC}_{10}(50 \mu \mathrm{M})$ and $\mathrm{IC}_{20}(100 \mu \mathrm{M})$ of CRYO resulted in a significant $(p<0.001)$ and synergistic increase of doxorubicin cytotoxicity of about 4- and 6-fold respectively (Figure 2B, Table I). Conversely, in CEM/ADR5000, the combination of doxorubicin with $\mathrm{IC}_{10}$ $(158 \mu \mathrm{M})$ and $\mathrm{IC}_{20}(200 \mu \mathrm{M})$ of CRYO (Figure 2C) determined an antagonistic effect, with a significantly reduction of doxorubicin $\mathrm{IC}_{50}$ of about 1.6- and 2.6-folds (Figure 2C; Table I).

Regarding CRY, a statistically significant $(p<0.001)$ decrease of doxorubicin $\mathrm{IC}_{50}$ value of about 4.5 fold was found only with the $\mathrm{IC}_{20}(644 \mu \mathrm{M})$ concentration in Caco-2 cell line. According to the combination index, the isobologram analysis showed a synergistic effect of the substance (Figure 3A, Table I). When tested in leukemic cells, in spite of lack chemosensitizing effects produced in the CCRF/CEM cells (Table I), the substance was able to increase the doxorubicin effectiveness in CEM/ADR5000. The $\mathrm{IC}_{50}$ value of the anticancer drug was reduced of about 1.5 - and 2-fold in the presence of $\mathrm{IC}_{10}(180 \mu \mathrm{M})$ and $\mathrm{IC}_{20}$ $(234 \mu \mathrm{M})$ concentrations of CRY, respectively (Figure 3B; Table I). The combination index and the isobologram analysis highlighted an antagonistic effect of CRY on doxorubicin.

Rhodamine 123 efflux assay. Both sesquiterpenes were able to increase the Rho123 content into the cells, likely due to the inhibition of the efflux pumps. In particular, in Caco-2 cells, CRYO produced a greater effect than the positive control verapamil $(20 \mu \mathrm{M})$ : starting from the concentration of $80 \mu \mathrm{M}$, the fluorescence increase was statistically significant and concentration-dependent (Figure 4A). In the same cell line, CRY showed a similar effect than verapamil at all tested concentrations (Figure 5A). Also in CEM/ADR5000 cells, both CRYO and CRY showed to inhibit the Rho123 efflux. However, while CRYO showed a similar potency than verapamil (Figure 4C) a lower effect 

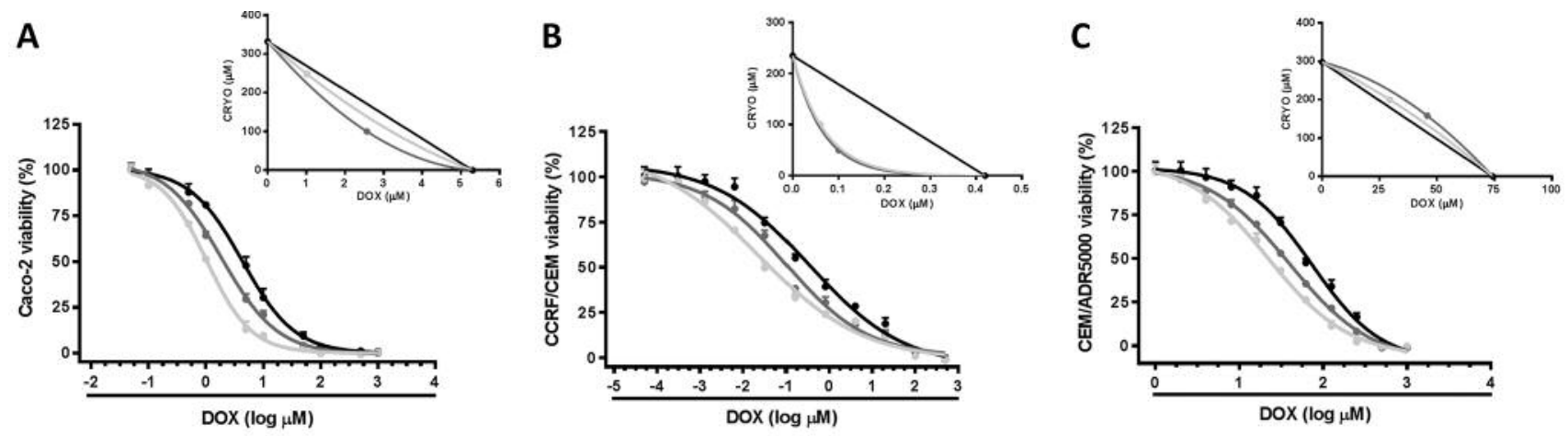

Figure 2. Concentration-response curves for the cytotoxicity of doxorubicin (DOX) alone (black line) and in combination with $\beta$-caryophyllene oxide (CRYO) at inhibitory concentration IC ${ }_{10}$ (dark gray line) and $I_{20}$ (light gray line) against Caco-2 (A), CCRF/CEM (B) and CEM/ADR5000 (C) cells. Also the isobologram analyses are reported for each concentration-response curves. In this analysis, the $I_{50}$ concentrations of DOX and CRYO are plotted on $x$ and $y$ axes, respectively. The line connecting these two points represents the additive effect; points located below and above the line indicate a synergistic and antagonistic effect, respectively. Values are expressed as the mean $\pm S E M(n=9)$.

Table I. Quantitative interaction of the combined treatment between $\beta$-caryophyllene $(C R Y)$ or $\beta$-caryophyllene oxide $(C R Y O)$, at IC ${ }_{10}$ and IC $C_{20}$ concentrations, and doxorubicin (DOX) in Caco-2, CCRF/CEM and CEM/ADR5000 cells.

\begin{tabular}{|c|c|c|c|c|c|c|}
\hline & \multicolumn{2}{|c|}{ Caco-2 } & \multicolumn{2}{|c|}{ CCRF/CEM } & \multicolumn{2}{|c|}{ CEM/ADR5000 } \\
\hline & $\begin{array}{c}\mathrm{IC}_{50}[\mu \mathrm{M}] \\
\left(\mathrm{RR}^{\mathrm{a}}\right)\end{array}$ & $\mathrm{CI}^{\mathrm{b}}$ & $\begin{array}{c}\mathrm{IC}_{50}[\mu \mathrm{M}] \\
\left(\mathrm{RR}^{\mathrm{a}}\right)\end{array}$ & $\mathrm{CI}^{\mathrm{b}}$ & $\begin{array}{c}\mathrm{IC}_{50}[\mu \mathrm{M}] \\
\left(\mathrm{RR}^{\mathrm{a}}\right)\end{array}$ & $\mathrm{CI}^{\mathrm{b}}$ \\
\hline DOX & $5.24 \pm 0.66$ & - & $0.42 \pm 0.02$ & - & $74.28 \pm 0.35$ & - \\
\hline $\mathrm{DOX}+\mathrm{CRY} \mathrm{IC}_{10}$ & $\begin{array}{c}3.71 \pm 0.05 \\
(-)\end{array}$ & - & $\begin{array}{c}0.35 \pm 0.005 \\
(-)\end{array}$ & - & $\begin{array}{c}47.23 \pm 0.86 \\
(1.6 \pm 0.1)\end{array}$ & $1.1 \pm 0.02$ \\
\hline $\mathrm{DOX}+\mathrm{CRY} \mathrm{IC}_{20}$ & $\begin{array}{c}1.15 \pm 0.003 \\
(4.5 \pm 0.2)\end{array}$ & $0.81 \pm 0.03$ & $\begin{array}{c}0.31 \pm 0.007 \\
(-)\end{array}$ & - & $\begin{array}{c}35.35 \pm 1.33 \\
(2.0 \pm 0.1)\end{array}$ & $1.1 \pm 0.02$ \\
\hline $\mathrm{DOX}+\mathrm{CRYO} \mathrm{IC}_{10}$ & $\begin{array}{c}2.57 \pm 0.04 \\
(2.0 \pm 0.1)\end{array}$ & $0.82 \pm 0.06$ & $\begin{array}{l}0.1 \pm 0.01 \\
(4.0 \pm 0.2)\end{array}$ & $0.49 \pm 0.03$ & $\begin{array}{c}45.79 \pm 1.12 \\
(1.6 \pm 0.1)\end{array}$ & $1.2 \pm 0.03$ \\
\hline $\mathrm{DOX}+\mathrm{CRYO}^{\mathrm{IC}} \mathrm{C}_{20}$ & $\begin{array}{l}1.02 \pm 0.06 \\
(4.7 \pm 0.3)\end{array}$ & $0.96 \pm 0.03$ & $\begin{array}{c}0.06 \pm 0.001 \\
(6.4 \pm 0.3)\end{array}$ & $0.58 \pm 0.01$ & $\begin{array}{c}29.44 \pm 0.70 \\
(2.6 \pm 0.2)\end{array}$ & $1.1 \pm 0.02$ \\
\hline
\end{tabular}

For each experiments, the technical replicates were averaged and the averages (expressed as mean \pm SEM) were evaluated by one-way ANOVA

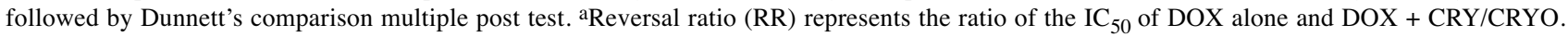
bCombination index (CI) is interpreted as follows: $<1$ synergism, $>1$ antagonism, and =1 additive.

was highlighted for CRY (Figure 5C). At last, in CCRF/CEM cell line, which does not express the ABC-transporters, Rho123 efflux was not affected by verapamil, CRYO and CRY (Figures 4B and B).

\section{Discussion}

Under our experimental conditions, CRY and CRYO have been assayed as chemosensitizing agents due to their low cytotoxicity. Accordingly, previous studies highlighted a weak antiproliferative activity for these compounds (9-11), although some cytotoxic effects were reported for CRYO $(12,13)$ : these conflicting results require to be better defined and may be a consequence of the low bioavailability of these sesquiterpenes in hydrophilic media (14).

When tested in combination experiments, CRY and CRYO were able to potentiate the doxorubicin cytotoxicity, although not always in a synergistic way. In particular, an antagonism was highlighted for both substances in the CEM/ADR5000 cell line: this effect could be due to the ratio between the combined agents, according to what previously reported (15). Nevertheless, both substances increased the doxorubicin cytotoxicity in a synergistic manner in Caco-2 and CCRF/CEM cells, being CRYO more effective than CRY. In order to evaluate if an impairment of the $\mathrm{ABC}$ transporter function could be involved in these chemosensitizing effects, 

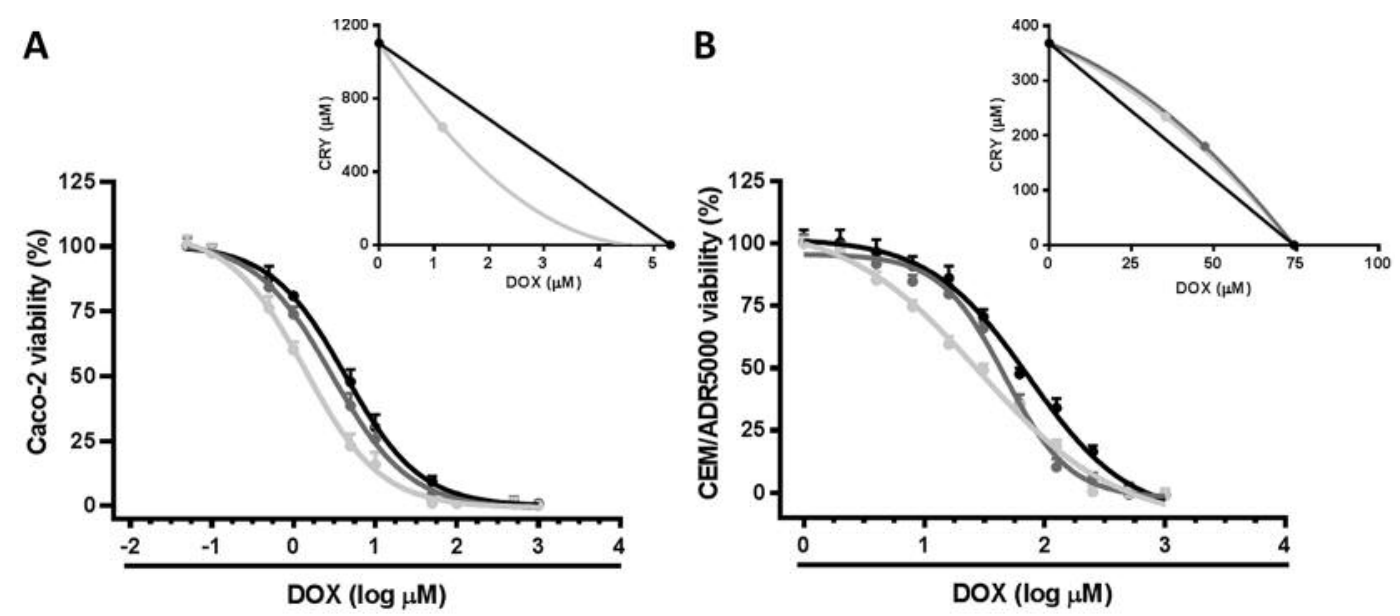

Figure 3. Concentration-response curves for the cytotoxicity of doxorubicin (DOX) alone (black line) and in combination with $\beta$-caryophyllene $(C R Y)$ at inhibitory concentration $I C_{10}$ (dark gray line) and $I C_{20}$ (light gray line) against Caco-2 (A) and CEM/ADR5000 (B) cells. Also the isobologram analyses are reported for each concentration-response curve. In this analysis, the $I_{50}$ concentrations of DOX and CRYO are plotted on $x$ and $y$ axes, respectively. The line connecting these two points represents the additive effect; points located below and above the line indicate a synergistic and antagonistic effect, respectively. Values are expressed as the mean $\pm S E M(n=9)$.

A

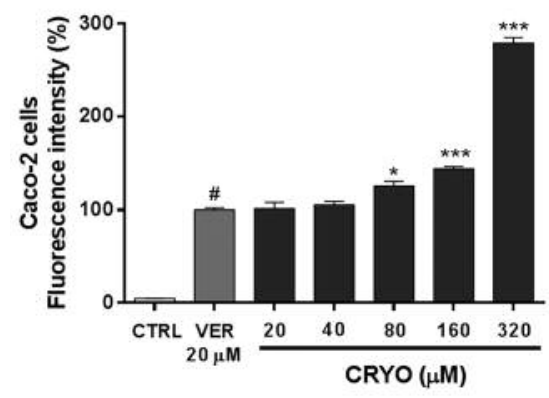

B

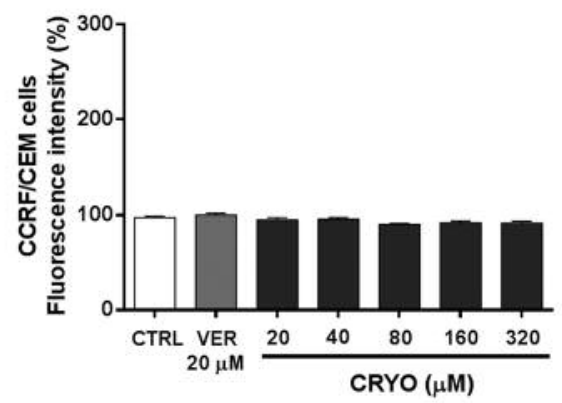

C

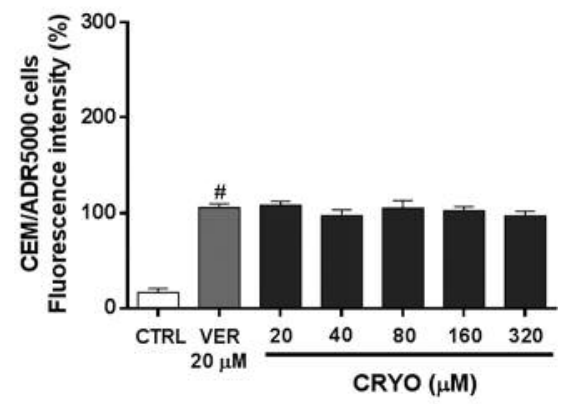

Figure 4. Effect of $\beta$-caryophyllene oxide (CRYO) on rhodamine 123 efflux in Caco-2 (A), CCRF/CEM (B) and CEM/ADR5000 (C) cells. Values are expressed as the mean $\pm \operatorname{SEM}(n=9)$. VER, Verapamil. Statistically significantly different at $* p<0.05$ and $* * * p<0.001$ from VER, and at ${ }^{\#} p<0.001$ from the negative control.

A

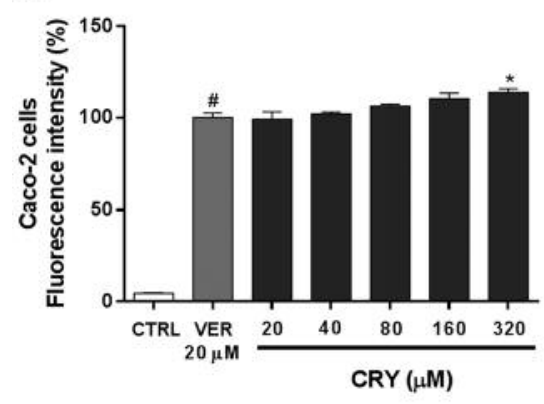

B

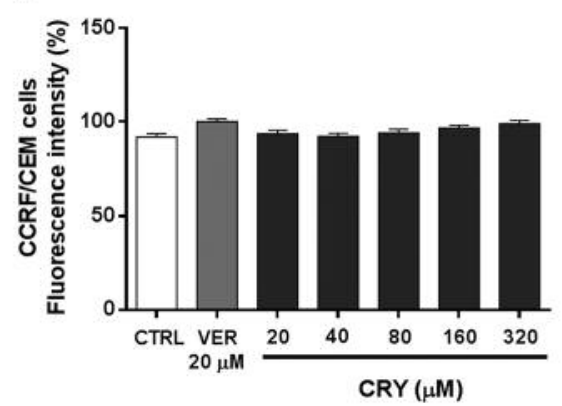

C

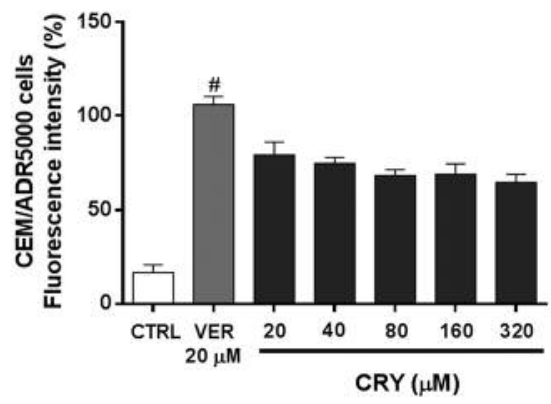

Figure 5. Effect of $\beta$-caryophyllene (CRY) on rhodamine 123 efflux in Caco-2 (A), CCRF/CEM (B) and CEM/ADR5000 (C) cells. Values are expressed as the mean $\pm S E M(n=9)$. VER, Verapamil. Statistically significantly different at $* p<0.05$ and $* * * p<0.001$ from VER, and at ${ }^{\#} p<0.001$ from the negative control. 
the rhodamine efflux assay was carried out. The role of specific efflux pumps was studied by comparing the behaviour of the cell lines, which are characterized by different transporters. Particularly, CCRF/CEM cells are lacking $\mathrm{ABC}$ transporters, Caco-2 is characterized by all Pgp, MRP1 and BCRP pumps, while CEM/ADR5000 cells express only P-gp.

Both sesquiterpenes were able to interfere with the $\mathrm{ABC}$ pump function, although CRYO was the most effective. Analogously, previous evidences highlighted that CRY inhibited the P-gp activity $(16,17)$, while CRYO was reported to enhance doxorubicin cytotoxicity by increasing its intracellular accumulation and the oxidative stress (11).

CRY and CRYO are structurally related sesquiterpenes, being CRYO the epoxide analogue of CRY (7). The greater impact of CRYO could be ascribed to the reactive epoxide function, which may lead to the alkylation and block of transporter proteins. Due to their hydrophobic character (particularly CRY), the substances have been found to deeply bind the phospholipids of a DMPC-MLV biomembrane model and to slowly diffuse across the bilayer (18). Lipophilic compounds are able to accumulate into biomembrane and to cause an apparent "expansion" of the surface area, due to the swelling of the phospholipid bilayer upon their accumulation. The partition coefficient of the compound is an important requirement for this interaction: a $\log p$-value equal to 2.92 or higher allows a hydrophobic/van der Waals interaction of the substance with the steroid-binding hydrophobic region (SBHR) of transporters (19). For CRY and CRYO a $\log p$-value of 4.319 and 4.165 is reported (20). This allows us to hypothesize that interacting with the biomembrane phospholipids, the sesquiterpenes lead to the alteration of the membrane permeability and the activity of the transmembrane proteins, including the ABC-transporters (21). Terpenoids are known to target the lipophilic core of proteins, so altering their interaction with phospholipids and their function; some of them are also P-gp inhibitors (22). Altogether these data allow us to hypothesize a potential role of CRY and CRYO as new chemosensitizing agents to use in combination with doxorubicin in order to overcome the MDR phenomenon. The use of these combinations could be useful to reduce doxorubicin-based therapy toxicity and prevent MDR development.

\section{Acknowledgements}

This work was supported by the Enrico and Enrica Sovena Foundation (Rome, Italy).

\section{References}

1 Zhao Y, Huan ML, Liu M, Cheng Y, Sun Y, Cui H, Liu DZ, Mei QB and Zhou SY: Doxorubicin and resveratrol co-delivery nanoparticle to overcome doxorubicin resistance. Sci Rep 6: 35267, 2016.
2 van de Ven R, Oerlemans R, van der Heijden JW, Scheffer GL, de Gruijl TD, Jansen G and Scheper RJ: ABC drug transporters and immunity: novel therapeutic targets in autoimmunity and cancer. J Leukoc Biol 86(5): 1075-1087, 2009.

3 Kunjachan S, Rychlik B, Storm G, Kiessling F and Lammers T: Multidrug resistance: Physiological principles and nanomedical solutions. Adv Drug Deliv Rev 65: 1852-1865, 2013.

4 Kathawala RJ, Gupta P, Ashby CR Jr. and Chen ZS: The modulation of $\mathrm{ABC}$ transporter-mediated multidrug resistance in cancer: a review of the past decade. Drug Resist Updat 18: 117, 2015.

5 Chou TC: Theoretical basis, experimental design, and computerized simulation of synergism and antagonism in drug combination studies. Pharmacol Rev 58: 621-681, 2006. Erratum in: Pharmacol Rev 59: 124, 2006.

6 Nobili S, Landini I, Mazzei T and Mini E: Overcoming tumor multidrug resistance using drugs able to evade P-glycoprotein or to exploit its expression. Med Res Rev 32(6): 1220-1062, 2012.

7 Di Giacomo S, Mazzanti G and Di Sotto A: Mutagenicity of cigarette butt waste in the bacterial reverse mutation assay: The protective effects of $\beta$-caryophyllene and $\beta$-caryophyllene oxide. Environ Toxicol 31(11): 1319-1328, 2016.

8 Di Giacomo S, Di Sotto A, El-Readi MZ, Mazzanti G and Wink M: $\alpha$-Hexylcinnamaldehyde Synergistically Increases Doxorubicin Cytotoxicity Towards Human Cancer Cell Lines. Anticancer Res 36(7): 3347-3351, 2016.

9 Kaneda N, Pezzuto JM, Kinghorn AD, Farnsworth NR, Santisuk T, Tuchinda P, Udchachon J and Reutrakul V: Plant anticancer agents, L. cytotoxic triterpenes from Sandoricum koetjape stems. J Nat Prod 55(5): 654-659, 1992.

10 Legault J, Dahl W, Debiton E, Pichette A and Madelmont JC: Antitumor activity of balsam fir oil: production of reactive oxygen species induced by alpha-humulene as possible mechanism of action. Planta Med 69: 402-407, 2003.

11 Ambrož M, Boušová I, Skarka A, Hanušová V, Králová V, Matoušková P, Szotáková B and Skálová L: The Influence of Sesquiterpenes from Myrica rubra on the Antiproliferative and Pro-Oxidative Effects of Doxorubicin and Its Accumulation in Cancer Cells. Molecules 20(8): 15343-15358, 2015.

12 Kubo I, Chaudhuri SK, Kubo Y, Sanchez Y, Ogura T, Saito T, Ishikawa $\mathrm{H}$ and Haraguchi $\mathrm{H}$ : Cytotoxic and antioxidative sesquiterpenoids from Heterotheca inuloides. Planta Med 62: 427-430, 1996.

13 Sibanda S, Chigwada G, Poole M, Gwebu ET, Noletto JA, Schmidt JM, Reac AI and Setzer WN: Composition and bioactivity of the leaf essential oil of Heteropyxis dehniae from Zimbabwe. J Ethnopharmacol 92: 107-111, 2004.

14 Jun NJ, Mosaddik A, Moon JY, Jang KC, Lee DS, Ahn KS and Cho SK: Cytotoxic Activity of $\beta$-Caryophyllene Oxide Isolated from Jeju Guava (Psidium cattleianum Sabine) Leaf. Rec Nat Prod 5: 242-246, 2011.

15 Harasym TO, Liboiron BD and Mayer LD: Drug ratio-dependent antagonism: a new category of multidrug resistance and strategies for its circumvention. Methods Mol Biol 596: 291-323, 2010.

16 Zhang W and Lim LY: Effects of spice constituents on Pglycoprotein-mediated transport and CYP3A4-mediated metabolism in vitro. Drug Metab Dispos 36(7): 1283-1290, 2008.

17 Legault $J$ and Pichette A: Potentiating effect of $\beta$-caryophyllene on anticancer activity of $\alpha$-humulene, isocaryophyllene and paclitaxel. J Pharm Pharmacol 59: 1643-1647, 2007. 
18 Sarpietro MG, Di Sotto A, Accolla ML and Castelli F: Interaction of $\beta$-caryophyllene and $\beta$-caryophyllene oxide with phospholipid bilayers: Differential scanning calorimetry study. Thermochimica Acta 600: 28-34, 2015.

19 Wang RB, Kuo CL, Lien LL and Lien EJ: Structure-activity relationship: analyses of p-glycoprotein substrates and inhibitors. J Clin Pharm Ther 28(3): 203-228, 2003.

20 Chang HJ, Kim HJ and Chun HS: Quantitative structure-activity relationship (QSAR) for neuroprotective activity of terpenoids. Life Sci 80(9): 835-841, 2007.
21 Sikkema J, de Bont JA and Poolman B: Interactions of cyclic hydrocarbons with biological membranes. J Biol Chem 269(11): 8022-8028, 1994.

22 Eid SY, El-Readi MZ and Wink M: Carotenoids reverse multidrug resistance in cancer cells by interfering with $\mathrm{ABC}$ transporters. Phytomedicine 19: 977-987, 2012.

Received December 28, 2016

Revised February 3, 2017

Accepted February 7, 2017 